\title{
Algunos factores relacionados con el trastorno por déficit de atención con hiperactividad en niños en edad escolar del distrito de Trujillo, Perú.
}

\author{
Attention deficit and hyperactivity disorder and some related factors in school children in Trujillo, Peru. \\ Benilde C. Tirado-Hurtado ${ }^{1}$, Cristopher Salirrosas-Alegría ${ }^{2}$, Lourdes Armas-Fava ${ }^{3}$, \\ Conchita Asenjo-Pérez ${ }^{4}$.
}

\section{RESUMEN}

Objetivo: Determinar los factores familiares y biológicos relacionados con el trastorno por déficit de atención con hiperactividad (TDAH) en escolares del distrito de Trujillo. Material y métodos:Se realizó un estudio transversal, con una población de escolares entre 6 y 12 años. El muestreo se realizó por medio de la estimación de proporciones; el diseño fue combinado entre el estratificado, el conglomerado y el aleatorio simple, con una muestra de 390 niños. Se aplicó un test diagnóstico a padres y maestros, además de investigarse los factores familiares y biológicos. Se utilizó la prueba no paramétrica Chi cuadrado con nivel de significancia de 0,05 para determinar la relación entre los factores y el trastorno. Resultados: La frecuencia del TDAH fue del 9,74\%, con predominio en el sexo masculino $(63,2 \%)$ y en el grupo etario entre 8 y 10 años (39,5\%). El TDAH tipo mixto fue el más frecuente. Los antecedentes psiquiátricos en la familia relacionados fueron hiperactividad, trastorno bipolar y esquizofrenia. Los factores biológicos relacionados fueron: amenaza de aborto (23\%), tabaquismo (7,8\%), prematuridad (13,2\%), bajo peso al nacer $(18,4 \%)$ y asfixia $(23,6 \%)$. Conclusiones: Los factores familiares relacionados con el TDAH fueron los antecedentes psiquiátricos; y los biológicos fueron amenaza de aborto, tabaquismo, prematuridad, bajo peso al nacer y asfixia.

PALABRAS CLAVE: Trastorno por déficit de atención con hiperactividad, niño, factores de riesgo.

\section{SUMMARY}

Objective: To determine family and biological factor related to attention deficit disorder with hyperactivity (ADHD) in school district of Trujillo. Methods: We performed a cross-sectional study, with a population of schoolchildren aged 6 to 12 years old. Sampling was done by estimating proportions; the design was combined between stratified, cluster and simple random, with a sample of 390 children. A diagnostic test was applied to parents and teachers, also family and biological factors were investigated. We used nonparametric chi-square with a significance level

Médica residente de Psiquiatría. Universidad Nacional Mayor de San Marcos. Hospital Víctor Larco Herrera. Lima, Perú.

2 Médico residente de Psiquiatría. Universidad Peruana Cayetano Heredia. Instituto Nacional de Salud Mental Honorio Delgado - Hideyo Noguchi. Lima. Perú.

3 Médica Internista. Magíster en Medicina. Hospital Belén de Trujillo. Facultad de Medicina. Universidad Nacional de Trujillo. Trujillo, Perú.

4 Médica Psiquiatra. Magíster en Medicina. Hospital Regional Docente de Trujillo. Facultad de Medicina. Universidad Nacional de Trujillo. Trujillo, Perú. 
of 0.05 to determine the relationship between the factors and the disorder. Results: The frequency of ADHD was $9.74 \%$, with predominance in males (63.2\%) and in the age group between 8 and 10 years old (39.5\%). ADHD combined type was the most frequent. Related psychiatric history was hyperactivity disorder, bipolar disorder and schizophrenia. Biological factors related were: threatened abortion (23\%), smoking (7.8\%), prematurity (13.2\%), low birth weight (18.4\%) and asphyxia (23.6\%). Conclusions: Family factors related to ADHD were psychiatric history, and biological factors were threatened abortion, smoking, prematurity, low birth weight and asphyxia.

KEY WORDS: Attention deficit disorder with hyperactivity, child, risk factors.

\section{INTRODUCCIÓN}

El Trastorno por déficit de atención con hiperactividad (TDAH) es una patología psiquiátrica muy frecuente durante el periodo escolar que se caracteriza por la presencia de tres síntomas nucleares: inatención, hiperactividad e impulsividad, que son más frecuentes y graves que los observados habitualmente en sujetos de un nivel de desarrollo similar; afectando el funcionamiento académico, social y laboral de quien lo padece.(1-6). Este trastorno representa un problema complejo debido a que influye en la vida diaria del niño teniendo amplias repercusiones en su desarrollo, capacidad de aprendizaje y ajuste social, además de su posible persistencia a lo largo de la vida (5-9).

Existe variación en la prevalencia del TDAH alrededor del mundo. En países latinoamericanos se han hallado prevalencias de TDAH de $23,5 \%$ en México (1) y del 15,8 al 20,4\% en Colombia (5), en niños de edad escolar.

El diagnóstico clínico del TDAH se basa en una historia clínica completa y en la observación directa de los padres, profesores y médicos especializados $(11,12)$. Para el diagnóstico del TDAH existen el sistema DSM IV (6) de la Asociación Americana de Psiquiatría y el Sistema CIE-10 de la Organización Mundial de la Salud (13), en ambos, existen diferencias entre sus criterios diagnósticos que hacen difícil comparar los resultados de los distintos estudios. Para diagnosticar un trastorno hipercinético (término con que denomina el CIE 10 al TDAH), según criterios CIE-10, se requiere que el paciente presente al menos síntomas persistentes de inatención (6 síntomas), de hiperactividad (tres síntomas) y de impulsividad (un síntoma) presentes en más de un ambiente de la vida del niño, especificando subtipos parciales si los síntomas son de una sola área; de tal manera que el CIE-10 define un síndrome más grave, por tanto menos frecuente que el DSM IV y que define al TDAH de una forma más amplia, requiere para el diagnóstico la presencia de síntomas de inatención (6 síntomas) o de hiperactividad/impulsividad(6 síntomas) para los tipos inatento o hiperactivo/impulsivo o síntomas en ambas áreas para el tipo combinado. Así, en el DSM IV, existe la probabilidad, no contemplada en el CIE -10, de que un niño con el tipo inatento pueda no tener un síntoma de hiperactividad/impulsividad. Por ello, si se siguen los criterios de la CIE-10, es probable que los niños con el tipo inatento se encuentren subdiagnosticados, produciéndose así un falso negativo. Por otro lado, al usar los criterios del DSM IV es más probable diagnosticar las formas de hiperactividad e inatención leves en el niño (teóricamente normales para la edad) como TDAH, con mayor riesgo de obtener falsos positivos en el diagnóstico (11)

La etiología del TDAH aún no se ha clarificado aunque se plantea una multicausalidad (7). Entre los hallazgos neurobiológicos se anotan disfunciones en las vías fronto-estriatales en relación a la actividad noradrenérgica y dopaminérgica $(6,12)$. Las investigaciones sugieren además la existencia de un factor hereditario; sin embargo, pese a esto se sabe que los factores ambientales son importantes en el mantenimiento y la evolución de los síntomas.

Entre los factores prenatales se enumeran el consumo de alcohol por la madre gestante, el tabaquismo y el uso de otras sustancias psicoactivas, y algunas infecciones virales (8,14-16). Entre los factores perinatales se mencionan la asfixia neonatal, el bajo peso al nacer, el parto pre-término, y complicaciones varias del parto (como hemorragias y preeclampsia), incluyendo las secuelas tempranas como las convulsiones neonatales. Otros factores asociados al TDAH, de tipo sociofamiliar, son el bajo nivel socioeconómico, bajo coeficiente intelectual y trastornos de conducta en los progenitores (16-17).

Adicionalmente, en el ámbito psicosocial se sabe que el niño con TDAH recibe constantemente respuestas negativas a su impulsividad e hiperactividad por parte de su entorno lo cual contribuye a su baja autoestima. En el plano académico, a pesar que la mayor parte de los niños con TDAH presentan un coeficiente intelectual similar al resto, se ha visto retraso académico e incapacidades específicas del 
aprendizaje (10-12,15). Existe además evidencia de una comorbilidad significativa (trastorno oposicionista desafiante, alteraciones de la conducta, trastornos del ánimo y trastornos de ansiedad, dislexia y trastorno de la Tourette) $(5,14,18)$ y peor funcionamiento en la edad adulta (15-60\%), afirmándose que el TDAH influye en el comportamiento violento del joven y el mayor riesgo de deserción escolar y drogadicción $(5,9)$. Finalmente, estudios longitudinales y recientes señalan que los niños con TDAH generan utilización de recursos médicos de más del doble que los niños de igual edad sin TDAH, lo que significa que la carga negativa del TDAH se extiende más allá de los parámetros sociales, conductuales y académicos (10).

Conociendo el gran impacto del TDAH en la sociedad y los escasos estudios publicados acerca de este problema en nuestro medio, el objetivo del presente trabajo fue identificar los factores familiares y biológicos relacionados con el trastorno por déficit de atención con hiperactividad (TDAH) en escolares del distrito de Trujillo.

\section{MATERIAL Y MÉTODOS}

Se realizó un estudio descriptivo analítico. La población objetivo estuvo compuesta por todos los escolares niños y adolescentes entre los 6 y 12 años de edad de todas las instituciones educativas del distrito de Trujillo en el año 2007. Se excluyeron a niños con trastornos fisiológicos (disfunción sensorial, hipoacusia, o trastornos visuales); niños con evidencias físicas de alteraciones genéticas y niños asmáticos que consumían corticoides y/o agonistas beta adrenérgicos. Para determinar el tamaño de muestra se partió del número total de estudiantes, 40005 escolares entre los 6 y 12 años de edad, que se matricularon en las instituciones educativas del distrito de Trujillo durante el año 2006 (19). El cálculo del tamaño de muestra se realizó aplicando la fórmula para estimación de proporciones, con un nivel de significancia de 0,05 $(Z=1,96)$ y una proporción estimada (p) de 0,5, resultando un número de 381 escolares. El diseño de muestra fue combinado entre el estratificado (tipo de colegio nacional y particular) y el conglomerado (cada centro educativo) en dos etapas (primera etapa incluyó colegios y segunda etapa las aulas) y el aleatorio simple (dentro de cada aula). La muestra constó de 13 colegios. Para modo de selección de los colegios, se tuvo en cuenta el tamaño del colegio de acuerdo

$$
n=\frac{z^{2} p q}{d^{2}+\frac{Z^{2} p q}{d^{2}}}
$$

al número de alumnos que tenía (conglomerados con probabilidades proporcionales al tamaño). El modo de selección de las secciones o aulas, fue el sistemático con arranque aleatorio, debido a que este método asegura la representatividad de la muestra. En cada aula se seleccionaron aleatoriamente 10 estudiantes.

Se utilizaron tres instrumentos de recolección de datos. El primero empleado para el diagnóstico de TDAH y los restantes para determinar los factores familiares y biológicos asociados al TDAH. En este último se incluyeron factores prenatales: amenaza de aborto, amenaza de parto prematuro, enfermedades durante el embarazo (rubéola, varicela, sífilis, toxoplasmosis), farmacodependencia (consumo de cocaína o marihuana), tabaquismo, embriaguez, hipertensión en el embarazo, edad de la madre durante el embarazo, consanguinidad; asimismo factores perinatales: lugar del parto, tipo de parto, prematuridad, bajo peso al nacer, asfixia al nacer, malformaciones congénitas; y finalmente factores postnatales: ictericia, hipoglucemia, convulsiones, transfusión sanguínea, enfermedad que necesito hospitalización, cirugía. Los maestros respondieron sólo el primer cuestionario, para cada uno de los alumnos de su grupo, mientras que los padres respondieron todos los cuestionarios.

El primer instrumento fue un cuestionario de lista de síntomas del TDAH del DSM-IV-TR, constaba de 21 preguntas. Las primeras 18 se refirieron a los síntomas de inatención, hiperactividad e impulsividad del criterio diagnóstico A del DSM-IV-TR. Cada síntoma se valoró en una escala Likert con cuatro grados de gravedad (nunca o casi nunca, algunas veces, muchas veces, siempre o casi siempre). Se calificaron las dimensiones de la lista de síntomas transformando las variables semicontinuas (grados de severidad) en categóricas; se otorgó un valor de cero a las preguntas contestadas como Nunca y algunas veces, suponiendo que los niños con esas puntuaciones no tendrían el síntoma. Asimismo, se otorgó un valor de uno a las preguntas contestadas como muchas veces y siempre o casi siempre, suponiendo que los sujetos con estas puntuaciones sí tendrían el síntoma correspondiente a la pregunta. Para diagnosticar TDAH se requirió un puntaje mínimo de 6 puntos tanto en la escala de desatención, como en la escala de hiperactividad impulsividad.

La pregunta 19 fue para indagar si la duración de los síntomas había sido de por lo menos 6 meses (parte del Criterio A del DSM- IV-TR). Las preguntas 20 y 21 fueron para establecer los criterios B (edad de inicio de 
los síntomas) y D (alteraciones que pudieran ocurrir en el rendimiento académico o en las relaciones sociales) del DSM-IV-TR. Estas tres últimas preguntas fueron contestadas en forma dicotómica: SÍ o NO.

\section{Procedimientos}

Previa recolección de información en los diferentes centros educativos seleccionados, se procedió a realizar un estudio piloto, cuyos datos sirvieron de base para la validación que se hizo del cuestionario de lista de síntomas del TDAH del DSM-IV-TR. En este proceso de validación, la medida de adecuación muestral de este test realizado a los padres y profesores fue de 0.748 (válido si la adecuación muestral es mayor de 0,5 ), con un coeficiente de fiabilidad o de confianza Alpha de Crombach's mayor que $0.7(0,824)$ que confirmaba su validez.

Se solicitó la autorización de las autoridades de los centros educativos y el consentimiento informado firmado por los padres de los niños que participaron en la investigación. Cuando una escuela o padre de familia se negaron a participar, se los reemplazó al azar por otra institución o persona dentro del mismo estrato (público o privado).

Posteriormente el diagnóstico de TDAH se hizo a los escolares que cumplían con los criterios diagnósticos mencionados, considerándose tanto la encuesta respondida por padres como profesores.

Los datos llenados en el cuestionario de factores biológicos fueron verificados mediante la revisión de las historia clínicas en las respectivas instituciones de salud donde fueron atendidas las madres y los niños. Finalmente, se alcanzaron los resultados del cuestionario de síntomas TDAH a los padres y a cada institución educativa, informándoseles si el test había resultado "Altamente positivo para el TDAH" o no. A los padres de aquellos niños cuyo resultado fue altamente positivo para TDAH se les brindó consejería y orientación para que buscasen ayuda de un especialista (psiquiatra).

\section{Análisis de Datos}

Para la presente investigación se utilizaron tablas de distribución de frecuencias uni y bidimensionales con sus valores absolutos y relativos. Se utilizó la prueba no paramétrica de independencia de criterios, usando la distribución Chi Cuadrado con un nivel de significancia de 0,05.Se empleó la corrección de Yates para aquellas frecuencias menores de 5 .

Además se utilizó el OR para el análisis de asociación de algunas variables (Tabla 4)

Todos los datos fueron procesados en el SPSS versión 14.0.

\section{Principios éticos}

El presente estudio fue aprobado por el Comité de Ética de la Facultad de Medicina de la Universidad Nacional de Trujillo.

\section{RESULTADOS}

En el presente trabajo se encontró una frecuencia de 38 niños con TDAH $(9,74 \%)$ sobre el total de escolares encuestados del distrito de Trujillo durante el año 2007 (Tabla 1). Del total de niños con TDAH, $24(63,2 \%)$ correspondieron al sexo masculino y $14(36,8 \%)$ al femenino, con una proporción de 1,7 varones con TDAH por cada mujer (Tabla 2).

En relación a los grupos etarios, la distribución más frecuente se encontró en el rango de 8 a 10 años con 15 casos (39,5\%) (Tabla 3$)$.

Tabla 1: Frecuencia del Trastorno por déficit de atención con hiperactividad en escolares del distrito de Trujillo. 2007

\begin{tabular}{ccc}
\hline & $\mathrm{n}$ & $\mathbf{\%}$ \\
\hline Niños con TDAH & 38 & $\mathbf{9 , 7 4}$ \\
Niños sin TDAH & 352 & $\mathbf{9 0 , 2 6}$ \\
Total & 390 & $\mathbf{1 0 0}$ \\
\hline
\end{tabular}

Fuente: Instrumento de recolección de datos.

Tabla 2: Distribución según sexo de la frecuencia del trastorno por déficit de atención con hiperactividad en escolares del distrito de Trujillo. 2007

\begin{tabular}{cccc}
\hline & \multicolumn{3}{c}{ TDAH } \\
\cline { 2 - 4 } & Sí n $(\%)$ & No n $(\%)$ & Total \\
\hline Masculino & $24(63,2)$ & $190(54,0)$ & 214 \\
Femenino & $14(36,8)$ & $162(46,0)$ & 176 \\
Total & $38(100,0)$ & $352(100,0)$ & 390 \\
\hline
\end{tabular}

Fuente: Instrumento de recolección de datos. 
Tabla 3: Distribución según edad de la frecuencia del trastorno por déficit de atención con hiperactividad en escolares del distrito de Trujillo. 2007.

\begin{tabular}{cccc}
\hline \multicolumn{4}{c}{ TDAH } \\
Edad & Si n $(\%)$ & No n $(\%)$ & Total \\
\hline $6-8$ & $13(34,2)$ & $144(40,9)$ & 157 \\
$8-10$ & $15(39,5)$ & $88(25,0)$ & 103 \\
$10-12$ & $10(26,3)$ & $120(34,1)$ & 130 \\
Total & $38(100,0)$ & $352(100,0)$ & 390 \\
\hline
\end{tabular}

Fuente: Instrumento de recolección de datos.

Tabla 4: Frecuencia del Trastorno por déficit de atención con hiperactividad distribuida según subtipo de acuerdo al grupo etáreo en escolares del distrito de Trujillo. 2007

\begin{tabular}{cccc}
\hline & Inatento & $\begin{array}{c}\text { Hiperactivo/ } \\
\text { Impulsivo }\end{array}$ & Combinado \\
\hline Edad & $\mathrm{n}(\%)$ & $\mathrm{n}(\%)$ & $\mathrm{n}(\%)$ \\
$6-8$ & $0(0,0)$ & $4(50,0)$ & $9(39,13)$ \\
$8-10$ & $4(57,1)$ & $3(37,5)$ & $8(34,78)$ \\
$10-12$ & $3(42,9)$ & $1(12,5)$ & $6(26,09)$ \\
Total & $7(100,0)$ & $8(100,0)$ & $23(100,0)$ \\
\hline
\end{tabular}

Fuente: Instrumento de recolección de datos.
De los subtipos de TDAH, 23 casos $(60,53 \%)$ correspondieron al subtipo combinado, seguido por el subtipo hiperactivo/impulsivo con 8 casos $(21,05 \%)$, y por el subtipo déficit de atención con 7 casos $(18,42 \%)$. El subtipo inatento fue más frecuente en el rango entre 8 y 10 años. Por su parte el tipo hiperactivo/impulsivo y el combinado se presentaron con mayor frecuencia en los niños entre 6 y 8 años (Tabla 4).

En la Tabla 5 se detallan los factores biológicos perinatales relacionados al TDAH que presentaron significación estadística además de un OR que señala que la presencia de estas variables aumenta el riesgo de presentar TDAH. Dichos factores biológicos fueron: amenaza de aborto, tabaquismo, prematuridad, bajo peso al nacer y asfixia neonatal.

\section{DISCUSIÓN}

Los resultados de este estudio muestran una frecuencia del TDAH del 9,74 \% en niños escolares, resultado similar al obtenido por Faraone $(3,6)$ quien señala una prevalencia a nivel mundial del TDAH entre el 8 y $12 \%$. Sin embargo dista de otros estudios latinoamericanos donde se llegan a reportar cifras de hasta $23,5 \%$ (1). Los intentos por determinar una tasa se ven afectados por toda una serie de factores $(1,2)$ : las variaciones en la clasificación clínica de los criterios diagnósticos (lo que ha afectado al número y a la combinación de signos necesarios para el diagnóstico del TDAH: DSM-III, DSM-IV, DSM-IV-TR, CIE 10), los métodos de evaluación diagnósticos empleados, la

Tabla 5: Factores biológico perinatales relacionados al Trastorno por déficit de atención con hiperactividad en los escolares del distrito de Trujillo. 2007

\begin{tabular}{|c|c|c|c|c|c|c|c|c|c|c|}
\hline \multirow{3}{*}{ Factor Perinatal } & \multicolumn{4}{|c|}{ TDAH } & \multirow{3}{*}{ Total } & \multirow{3}{*}{$\mathrm{x}^{2}$} & \multirow{3}{*}{$p$-value } & \multirow{2}{*}{\multicolumn{3}{|c|}{$\begin{array}{l}\text { Odd Ratio } \\
\text { Intervalo al } 95 \% \\
\end{array}$}} \\
\hline & \multicolumn{2}{|c|}{$\mathrm{Si}$} & \multicolumn{2}{|c|}{ No } & & & & & & \\
\hline & $\mathrm{n}$ & $\%$ & $\mathrm{n}$ & $\%$ & & & & Valor & Inferior & Superior \\
\hline $\begin{array}{l}\text { Amenaza de } \\
\text { aborto }\end{array}$ & 9 & 23.68 & 16 & 4.545 & 25 & 20,94 & 0,000005 & 6.517 & 2.649 & 16.037 \\
\hline $\begin{array}{l}\text { Tabaquismo } \\
\text { materno }\end{array}$ & 3 & 7.89 & 2 & 0.560 & 5 & $9,496^{*}$ & $<0.01$ & 15.21 & 2.459 & 94.143 \\
\hline Prematuridad & 5 & 13.16 & 17 & 4.802 & 22 & 4,46 & 0,034 & 4.523 & 1.041 & 8.663 \\
\hline $\begin{array}{l}\text { Bajo peso al } \\
\text { nacer }\end{array}$ & 7 & 18.42 & 26 & 7.407 & 33 & 5,39 & 0,020 & 2.823 & 1.134 & 7.028 \\
\hline Asfixia neonatal & 7 & 18.42 & 23 & 6.497 & 30 & 6,82 & 0,008 & 3.25 & 1.292 & 8.176 \\
\hline
\end{tabular}

(*): Se aplicó la corrección de Yates

Fuente: Instrumento de recolección de datos. 
fuente de información (padres, maestros, cuidadores), el tipo de muestra (clínica, poblacional), la práctica de pruebas de inteligencia como variable de control, y las características socioculturales $(2,5)$.

En cuanto a las diferencias por género, en el presente estudio se encontró que $63,2 \%$ de niños presentaba criterios para el TDAH frente a un $36,8 \%$ encontrado en las niñas. Cornejo y col (5) en Colombia encontraron una razón de prevalencia de 3,88 hombres por cada mujer, similar a la descrita por el DSM IV, 9:1 en la población clínica y 4:1 en la población normal (8). Por otro lado, Bauermeister y col encontraron diferencias en la prevalencia del TDAH respecto al género, solo cuando los subtipos fueron considerados, reportando que el subtipo combinado es más común en varones, mientras que el subtipo déficit de atención predomina en mujeres (20). Sin embargo estas diferencias parecen desaparecer en los adolescentes, con relaciones cercanas 1:1. En este sentido, en algunas revisiones, se ha apuntado que el hecho de no utilizar criterios diferenciales por género favorece un infradiagnóstico de las niñas (8-21), sugiriendo que las niñas con TDAH son una "silenciosa minoría" hasta que sus síntomas de hiperactividad y agresión no sean tan floridos como lo de los varones, hecho que ocurre durante la adolescencia (12). Por otra parte, Biederman y Faraone afirman que la diferencia en la relación referente al sexo probablemente se deba a que el TDAH causaría menos daño en la mujer que en el varón (3).

El subtipo más frecuente fue el combinado (hiperactividad y déficit de atención) el cual se relaciona a un mayor riesgo de desajustes en el desarrollo personal y social a largo plazo $(5,16,21)$. Al relacionar los subtipos con la edad, se evidencia que el subtipo combinado hiperactividad/impulsividad predomina en los primeros años, mientras que el subtipo inatento predomina en los niños de mayor edad, postulándose que la sintomatología motora del TDAH disminuye con la edad, mientras que el tipo cognitivo-atencional tiende a mantenerse (8).

Los resultados de este estudio nos permiten plantear la hipótesis de probables asociaciones entre la existencia de factores perinatales y las manifestaciones del TDAH. Barkley $(21,22)$ apoya nuestros resultados al afirmar que las madres que presentan complicaciones durante el embarazo o en el parto tienen mayor probabilidad de tener un niño con TDAH. En el estudio de Pineda y cols. (23), los factores de riesgo perinatales más frecuentes fueron: amenaza de aborto (18\%), amenaza de parto prematuro (20\%), hospitalización durante los primeros meses de vida $(17,2 \%)$, gripe $(17,1 \%)$, consumo de cigarrillos $(7 \%)$ y consumo de alcohol (4,7\%). Sin embargo en otro estudio de Montiel-Nava y cols. (3), el 82,5\% de las madres de niños con TDAH no presentó dificultades o complicaciones perinatales. Los problemas durante la gestación pueden causar TDAH al interferir en el desarrollo normal del feto (21); paralelamente existen otros niños que presentan un normal desarrollo en esa misma etapa, y que presentan hiperactividad durante la infancia (24).

De los factores informados, los que estuvieron más relacionados fueron la amenaza de aborto y el tabaquismo durante la gestación. La frecuencia de tabaquismo en madres $(7,8 \%)$ con niños con diagnóstico de TDAH fue altamente significativa, coincidiendo con Flores y cols. (16), en su estudio realizado en Perú en niños con diagnóstico clínico de TDAH, donde el tabaquismo materno representaba el $7,69 \%$ de los factores de riesgo biológicos prenatales asociados. En un estudio se encontró que los niños con TDAH se expusieron 2-2,5 veces más al consumo de cigarrillo y alcohol por parte de sus madres que los niños controles $(23,25)$. Según estudios realizados en roedores, los receptores nicotinícos modularían la actividad dopaminérgica, cuya alteración podría originar el TDAH (3), lo que sugiere que la nicotina podría dañar el cerebro en puntos críticos de su desarrollo. Por otro lado Fryer y cols. (26) encontraron que el sistema de control de la inhibición del circuito frontoestriatal sería sensible a la teratogénesis producida por el alcohol. Estos datos confirman la hipótesis de que el uso de sustancias tóxicas para el cerebro fetal se asocia con alteraciones en su maduración, que se pueden manifestar en forma de síntomas de diversos tipos de psicopatología infantil, incluyendo al TDAH (27).

Respecto a la farmacodependencia (consumo de marihuana o cocaína) durante la gestación, no se encontró diferencia significativa. Estos resultados coinciden con los reportados por Pineda y cols. quienes tampoco encontraron diferencias significativas entre los casos y controles (23).

Se encontró una diferencia estadísticamente significativa entre prematuridad y TDAH $(13,2 \%$ de los niños con TDAH frente a $4,8 \%$ de los niños sin TDAH). Al respecto Flores y cols. (16) encontraron cifras similares, reportando la prematuridad en 6,67\% de los niños con TDAH. Poeta y cols. (21) encontraron 
que $19 \%$ de niños con TDAH nacieron prematuros, a diferencia del estudio de Montiel-Nava y col (28) donde la prematuridad solo se observó en un caso $(2,5 \%)$ de los 40 niños con TDAH.

Referente al hallazgo en el presente estudio de una diferencia estadísticamente significativa entre el bajo peso al nacer y el TDAH $(18,4 \%$ de niños con TDAH con bajo peso al nacer frente a 7,3\% en niños sin TDAH), Indredavik y cols. encontraron que $25 \%$ de adolescentes con antecedentes de bajo peso al nacer presentaban síntomas de déficit de atención con hiperactividad, hallazgo similar a otros estudios que reportan cifras entre 5 y $32 \%$ (29). Sin embargo las causas estos hallazgos aún no son del todo claras.

En cuanto al antecedente de asfixia neonatal, el $18,4 \%$ de los niños con TDAH presentó asfixia al nacer, frente a $6,5 \%$ de niños sin $\mathrm{TDAH}$, existiendo una diferencia significativa entre ambos grupos. Flores y col encontraron que la hipoxia representaba el $80 \%$ de los factores de riesgo biológicos natales asociados al TDAH. Esta relación se explicaría en que el mecanismo común a los factores biológicos, en la génesis del TDAH, sería la hipoxia, lo que incrementaría los niveles de lactato e interferiría con los circuitos frontoestriatales en un momento crítico del desarrollo lo que a su vez condicionaría disturbios en el comportamiento, impulsividad, inatención e hiperactividad (16). Esto fue corroborado por Astbury y cols., en un estudio de seguimiento hasta los 5 años a niños con antecedentes de prematuridad e hipoxia al nacer, donde reportaron déficit de atención en el 34\% de su muestra (16).

\section{CONCLUSIONES}

- La frecuencia del TDAH en los escolares del distrito de Trujillo fue del 9,74\%, es decir que a nivel de la población debemos esperar entre 2719 y 5073 escolares con TDAH, predominando el sexo masculino y el grupo etario entre 8 y 10 años.

- El subtipo de TDAH más frecuente fue el combinado.

- Los factores biológicos relacionados con el TDAHque presentaron diferencia estadísticamente significativa en escolares en el distrito de Trujillo fueron: la amenaza de aborto, el tabaquismo materno, la prematuridad, el bajo peso al nacer y la asfixia.

\section{Correspondencia:}

Cristopher Salirrosas Alegría

Instituto Nacional de Salud Mental Honorio Delgado - Hideyo Noguchi.

Av. Eloy Espinoza 709. Urb. Palao. San Martín de Porres. Lima 31. Lima, Perú.

Teléfono: 6149200

Correo electrónico: csalirrosas@yahoo.com

\section{Conflictos de intereses:}

Ninguno

\section{REFERENCIAS BIBLIOGRÁFICAS}

1. Rivera L, Martínez R, Ortiz A, Ramírez A, Puente C, Ramírez M, et al. Prevalencia del trastorno de déficit de atención e hiperactividad en niños de edad preescolar en San Luis Potosí. Rev Mex Neuroci. 2004; 5(5): 462-468.

2. Cardo P, Servera M. Trastorno por déficit de atención con hiperactividad: Una visión global. An Pediatr. 2003; 59: 225-8.

3. Biederman J, Faraone S. Attention-deficit hyperactivity disorder. Lancet. 2005; 366: 237 - 48.

4. Swanson J, Sergeant J, Taylor E, Sonuga-Barke E, Jensen P, Cantwell D. Trastorno por déficit de atención con hiperactividad y trastorno hipercinético. Lancet. 1998;32(6):360-5

5. Cornejo J, Osío O, Sánchez Y, Carrizosa J, Sánchez $\mathrm{G}$, Grisales $\mathrm{H}$, et al. Prevalencia del trastorno por déficit de atención e hiperactividad en niños y adolescentes colombianos. Rev Neurol. 2005; 40: 716-22.

6. American Psychiatric Association. Diagnostic and statistical manual of mental disorders. Fourth edition - Text Revision (DSM-IV-TR). Washington DC: Masson; 2000.

7. Garduño F. Trastorno por déficit de atención e hiperactividad. Rev Mex Pediatr. 2003; 70: 87-90.

8. Cardo P, Servera-Barceló M. Prevalencia del trastorno por déficit de atención e hiperactividad. Rev Neurol. 2005; 40 (S1): S11-S15.

9. Goodman D. The consequences of attention-deficit/ hyperactivity disorder in adults. J Psychiatr Pract. 2007; 13: 318-27.

10. Ruiz M, Gutiérrez J, Garza S, De la Peña F. Trastorno por déficit de atención con hiperactividad: Actualidades diagnósticas y terapéuticas. Bol Med Hosp Infant Mex. 2005; 62: 145-152.

11. Soutullo C. Diagnóstico y tratamiento farmacológico del trastorno por déficit de atención e hiperactividad. Med Clin (Barc). 2003; 120(6):222-226.

12. Higuera F, Pella V. Trastorno por déficit de atención con hiperactividad: Revisión de conocimientos actuales. Acta Médica Grupo Ángeles. 2004; 2: 37-44 
13. Organización Mundial de la Salud. The ICD -10 classification of mental and behavioural disorders: clinical description and diagnosis guidelines 1992; diagnostis criteria for research 1993. Ginebra: WHO.1993. Disponible desde: http://www.who.int/ classifications/icd/en/GRNBOOK.pdf.

14. Faraone S, Sergeant J, Gillberg C, Biederman J. The worldwide prevalence of ADHD: is it an American condition? World Psych. 2003; 2: 104-113.

15. Brasset-Harknett A, Butler N. Attention-deficit/ hyperactivity disorder: An overview of the etiology and a review of the literature relating to the correlates and life course outcomes for men and women. Clin Psych Rev. 2005; 27: 188-210.

16. Flores A, Montenegro A. Factores de riesgo biológico asociado a niños con trastorno por déficit de atención e hiperactividad. Tesis de especialidad. Lima, Perú. Universidad Nacional Mayor de San Marcos, 2002.

17. Cantwell D. Attention deficit disorder: a review of the past 10 years. J Am Acad Child Adolesc Psychiatry. 1996; 35(8): 978-987.

18. Daley D. Attention deficit hyperactivity disorder: a review of the essential facts. Child Care Health Dev.2006; 32(2): 193-204.

19. Dirección Regional de Educación La Libertad. 2006. Disponible en: http:/www.drelalibertad.gob.pe/ (Fecha de acceso: enero del 2012).

20. Babinski LM, Hartsough CS, Lambert NM. Childhood conduct problems, hyperactivity/ impulsivity and inattention as predictors of adult criminal activity. J Child Psychol Psychiatry. 1999; 40: 347-55.

21. Poeta L, Rosa-Neto F. Características biopsicosociales de los escolares con indicadores de trastorno de déficit de atención e hiperactividad. Rev Neurol. 2006; 43 (10): 584-588.

22. Barkley R. Trastorno de déficit de atencao/ hiperactividade (TDAH): guia completo e autorizzado para os pais, profesores e profissionais de sáude. Porto Alegre: Artmed; 2002.

23. Pineda D, Puerta I, Merchán V, Arango C, Galvis A, Velásquez B, et al. Factores perinatales asociados con la aparición del trastorno por deficiencia de atención en niños de la comunidad colombiana "paisa". Rev Neurol. 2001; 32 (3):1-6.

24. Barbosa A. Hiperactividade em meninas: un estudio de prevalencia e diagnóstico. Arq Neuropsiquiatr. 2001; 59: S130-1.

25. Mick E. Biederman J, Faraone S, Sayer J, Kleinman S. Case-control study of deficit hyperactivity disorder and maternal smoking, alcohol use, and drug use during pregnancy. J Am Acad Child Adolesc Psychiatry. 2002; 41: 378-85.

26. Fryer S, Tapert S, Mattson S, Paulus M, Spadoni A, Riley E. Prenatal alcohol exposure affects frontalstriatal BOLD response during inhibitory control. Alcohol Clin Exp Res. 2007; 31: 1415-24.

27. Milberger S, Biederman J, Faraone SV, Jones J. Further evidence of an association between maternal smoking during pregnancy and attention deficit hyperactivity disorder: findings from a high-risk sample of siblings. J Clin Child Psychol. 1998; 27: 352-8.

28. Montiel-Nava C, Peña JA, Montiel- Barrero I. Datos epidemiológicos del trastorno por déficit de atención con hiperactividad en una muestra de niños marabinos. Rev Neurol. 2003;37(9): 815-9.

29. Indredavik M, Vik T, Heyerdahl S, Kulseng S, Fayers P, Brubakk A-M. Psychiatric symptoms and disorders in adolescents with low birth weight. Arch Dis Child Fetal Neonatal. 2004; 89: F445-F450.

30. Taylor E, Sandberg S, ThorleyG, Giles S. The epidemiology of childhood hyperactivity. London: Oxford University Press; 1992.

31. Milberger S, Biederman J, Faraone SV, Guite J, Tsuang MT. Pregnancy, delivery and infancy complications and attention deficit hyperactivity disorder: issues of gene-environment interaction. Biol Psychiatry. 1997; 53: 65-75.

32. Biederman J, Milberger S, Faraone V, Kiely K, Guite J, Mick E, et al. Family-environment risk factors for attention-deficit hyperactivity disorder. A test of Rutter's indicators of adversity. Arch Gen Psychiatry. 1998; 59 (S7): 4-16. 